\title{
Melodie Olson Responds
}

To the Editor:

I appreciate Melvin Morse's kind words about my study, and his interest in more data. I can supply answers for some of his questions.

1. Diagnoses on all patients were recorded from patients' charts. As few patients have only one diagnosis, the primary diagnosis as well as up to three secondary diagnoses were recorded. But diagnoses changed from the time of admission to discharge for many patients. Since data collectors were dependent on records currently in use and undergoing constant change, the accuracy of the diagnoses at any given time could not be guaranteed. Statistical correlations between group (i.e., OBEr vs. non OBEr) and type of patient (i.e., medical vs. surgical) were not significant. These correlations were only done on primary diagnoses. It should be remembered that 
many persons reported OBEs that occurred prior to the present hospitalization, some of them completely independent of any hospitalization. For those, anesthetic agents and the like are not confounding variables.

2. \& 5. All patients interviewed were alert and able to answer standard orientation questions. We listed medications for each interviewee. We then classified those medications that affect the central nervous system, and narcotics, separately. We noted whether any of those drugs had been taken within six hours of the interview. No relationships were found between reports of OBEs and use of these medications. In addition, the six patients who reported OBEs during this hospitalization were not taking any of the same drugs.

We did not attempt to classify severity of illness at this time.

3. This study focused on the OBE, not the NDE. People who report OBEs frequently have them within the context of an NDE. For our purposes, then, it was useful to consider the NDE as one context or set in which OBEs occur. You will notice that the way the question about OBEs is asked ("Have you ever felt your mind, consciousness, or center of awareness to be at a place different from your physical body?") will not elicit a positive response from NDErs who did not have an OBE as part of that experience. The statistics on OBEs are not reported as if they represent all NDEs that might have occurred.

In our sample of 31 reported OBEs, nine were associated with NDEs, 16 were not associated with NDEs, and in six cases that determination could not be made from the interview.

4. No data were kept related to patients who were not a part of the study, although I agree it would be helpful. Few patients actually refused participation, but many were off the care unit for tests or procedures, or had visitors and did not want to be interrupted while the data collectors were available. Because of short hospital stays, many of the patients were never asked to become a part of the study. One suggestion to avoid this problem in future studies is to work from admission lists and make appointments daily, at the time of admission and at discharge. That would require notification of all impending discharges, a luxury we did not have during this study. 
I appreciate the opportunity to repsond to these questions. Dialogue enriches the research process. I wish Morse good luck in his pursuit of greater empiricism in near-death research.

Melodie Olson, Ph.D., R.N. Associate Professor College of Nursing Graduate Program Medical University of South Carolina 171 Ashley Avenue Charleston, SC 29425-2404 